University of Wollongong

Research Online

Faculty of Engineering and Information

Faculty of Engineering and Information

Sciences - Papers: Part A

Sciences

$1-1-2010$

\title{
Femtosecond second-harmonic generation in periodically poled lithium niobate waveguides written by femtosecond laser pulses
}

\author{
Zhangchao Huang \\ Nankai University \\ Chenghou Tu \\ Nankai University \\ Shuanggen Zhang \\ Tianjin University of Technology \\ Yongnan Li \\ Nankai University \\ Fuyun Lu \\ Nankai University
}

See next page for additional authors

Follow this and additional works at: https://ro.uow.edu.au/eispapers

Part of the Engineering Commons, and the Science and Technology Studies Commons

Research Online is the open access institutional repository for the University of Wollongong. For further information contact the UOW Library: research-pubs@uow.edu.au 


\title{
Femtosecond second-harmonic generation in periodically poled lithium niobate waveguides written by femtosecond laser pulses
}

\author{
Abstract \\ We present in this Letter the second-harmonic generation of femtosecond pulses in double-line-written \\ waveguides fabricated in periodically poled lithium niobate (PPLN) with femtosecond laser pulses. In a \\ $10-\mathrm{mm}$-long sample, a normalized conversion efficiency of $12.6 \% \mathrm{~W}-1 \mathrm{~cm}-2$ has been achieved for $40 \mathrm{fs}$ \\ pump pulses with the wavelengths centered at $1550 \mathrm{~nm}$. Simulation results show that in PPLN \\ waveguides the FWHM of wavelength tuning curve for $40 \mathrm{fs}$ pump pulses is $42 \mathrm{~nm}$, which is 15 times of \\ that for 40 ps pump pulses.

\section{Keywords} \\ second, harmonic, femtosecond, laser, written, pulses, waveguides, generation, niobate, lithium, poled, \\ periodically \\ Disciplines \\ Engineering | Science and Technology Studies

\section{Publication Details} \\ Huang, Z., Tu, C., Zhang, S., Li, Y., Lu, F., Fan, Y. \& Li, E. (2010). Femtosecond second-harmonic generation \\ in periodically poled lithium niobate waveguides written by femtosecond laser pulses. Optics Letters, 355 \\ (11), 1752-1754.
}

\section{Authors}

Zhangchao Huang, Chenghou Tu, Shuanggen Zhang, Yongnan Li, Fuyun Lu, Yaxian Fan, and Enbang Li 


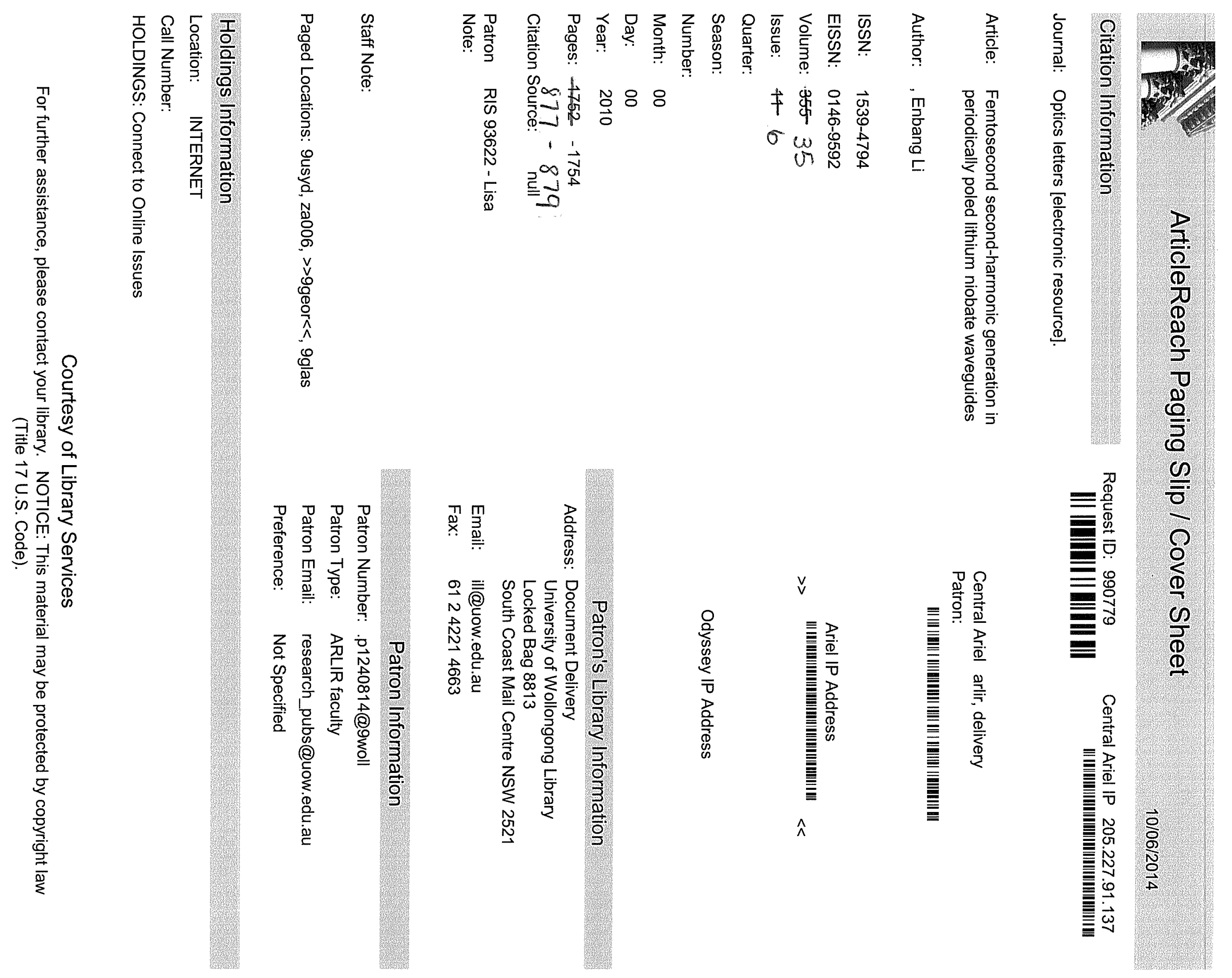




\title{
Femtosecond second-harmonic generation in periodically poled lithium niobate waveguides written by femtosecond laser pulses
}

\author{
Zhangchao Huang, ${ }^{1}$ Chenghou Tu, ${ }^{1}$ Shuanggen Zhang, ${ }^{2}$ Yongnan Li, ${ }^{1}$ Fuyun Lu, ${ }^{1, *}$ \\ Yaxian Fan, ${ }^{3}$ and Enbang $\mathrm{Li}^{4}$ \\ ${ }^{1}$ School of Physics, Nankai University, Tianjin 300071, China \\ ${ }^{2}$ School of Electronic Information Engineering, Tianjin University of Technology, Tianjin 300384, China \\ ${ }^{3}$ Department of Physics, Nanjing University, Nanjing 210093, China \\ ${ }^{4}$ College of Precision Instrument and Optoelectronics Engineering, Tianjin University, Tianjin 300072, China \\ *Corresponding author: lufy@nankai.edu.cn
}

Received October 26, 2009; revised January 9, 2010; accepted January 27, 2010; posted February 19, 2010 (Doc. ID 119068); published March 15, 2010

\begin{abstract}
We present in this Letter the second-harmonic generation of femtosecond pulses in double-line-written waveguides fabricated in periodically poled lithium niobate (PPLN) with femtosecond laser pulses. In a 10mm-long sample, a normalized conversion efficiency of $12.6 \% \mathrm{~W}^{-1} \mathrm{~cm}^{-2}$ has been achieved for $40 \mathrm{fs}$ pump pulses with the wavelengths centered at $1550 \mathrm{~nm}$. Simulation results show that in PPLN waveguides the FWHM of wavelength tuning curve for $40 \mathrm{fs}$ pump pulses is $42 \mathrm{~nm}$, which is 15 times of that for $40 \mathrm{ps}$ pump pulses. (C) 2010 Optical Society of America

OCIS codes: $160.4330,140.7090,140.3515$.
\end{abstract}

Nowadays quasi-phase-matching (QPM) waveguides in periodically poled materials have become one of the best choices for frequency doubling. To fabricate channel waveguides in transparent materials, several methods have been developed, such as Ti diffusion, annealed proton exchange, $\mathrm{Zn}$ diffusion, and femtosecond pulse writing. In the past decade there has been a rapid development of a femtosecond pulse writing method [1-7], owing to its simple fabrication process, fast realization of three-dimensional (3D) structure, and low cost. Recently more interests have arisen on second-harmonic generation (SHG) using laser beam written waveguides. Several groups [3-7], including ours, have investigated SHG characteristics in the waveguides for cw, nanosecond pulses and picosecond pulses. To the best of the authors' knowledge, however, investigations for the transmission of femtosecond pulses in these structures are still missing.

In this Letter, we describe the frequency doubling of femtosecond pulses in laser-written periodically poled lithium niobate (PPLN) waveguides with a QPM period of $18.6 \mu \mathrm{m}$. The experimental results show a second-harmonic ( $\mathrm{SH})$ spectrum width of 4.2 $\mathrm{nm}$ and provide the highest normalized conversion efficiency $12.6 \% \mathrm{~W}^{-1} \mathrm{~cm}^{-2}$ in the waveguides fabricated by femtosecond laser pulses.

To describe the evolution of ultrashort pulses' SHG, assuming the slowly varying envelope approximation and neglecting the losses, we use the coupled partial differential equations [8],

$$
\begin{aligned}
\frac{\partial E_{1}}{\partial z}-\frac{i}{2} \beta_{21} \frac{\partial^{2} E_{1}}{\partial \tau^{2}}= & -i \kappa d_{\mathrm{eff}} E_{2} E_{1}^{*} \exp (-i \Delta k z) \\
& -i \frac{n_{2} k_{1}}{n_{01}}\left(\left|E_{1}\right|^{2}+2\left|E_{2}\right|^{2}\right) E_{1},
\end{aligned}
$$

0146-9592/10/060877-3/\$15.00

$$
\begin{gathered}
\frac{\partial E_{2}}{\partial z}+\left(\frac{1}{v_{2}}-\frac{1}{v_{1}}\right) \frac{\partial E_{2}}{\partial \tau}-\frac{i}{2} \beta_{22} \frac{\partial^{2} E_{2}}{\partial \tau^{2}}=-i \kappa d_{\mathrm{eff}} E_{1}^{2} \\
\times \exp (-i \Delta k z)-i \frac{n_{2} k_{2}}{n_{02}}\left(\left|E_{2}\right|^{2}+2\left|E_{1}\right|^{2}\right) E_{2}
\end{gathered}
$$

where the indices 1 and 2 respectively represent fundamental and $\mathrm{SH}$ pulses, $i$ refers to either the indices 1 or $2, E_{i}$ is the field amplitude, $\kappa$ $=\left(2 w^{2} / \varepsilon_{0} c^{3} n_{01}{ }^{2} n_{02} S_{\text {eff }}\right)^{1 / 2}$ is the coupling coefficient, $n_{0 i}$ is the refractive index, $n_{2}$ is the third-order nonlinear refractive index, $\nu_{i}$ is the group velocity, and $\beta_{2 i}$ is the group-velocity dispersion parameter. Here, $\tau=t-z / v_{1}$ corresponds to a frame of reference moving with the fundamental pulse at its group velocity $v_{1}$. $\Delta k=k_{2}-2 k_{1}-2 \pi / \Lambda$ ( $\Lambda$ is the period of PPLN, and $k_{i}$ is the wave vector) is the wave-vector mismatch. The shape of the input pulse is of a standard Gaussian form. For a PPLN waveguide, the effective nonlinear coefficient $d_{\text {eff }}$ for first order QPM is $d_{\text {eff }}=2 d_{33} / \pi$ $=16 \mathrm{pm} / \mathrm{V}$, and the effective waveguide cross section is $S_{\text {eff }}=2.5 \times 10^{-10} \mathrm{~m}^{2}$. The walk-off length can be expressed as $L_{w}=T_{0} /\left(1 / v_{2}-1 / v_{1}\right)\left(T_{0}\right.$ is the width of fundamental pulse).

In Fig. 1(a), we calculated the normalized SHG efficiency for $40 \mathrm{fs}$ pump pulses with different central wavelengths in a PPLN waveguide with a fixed period of $18.6 \mu \mathrm{m}$ designed for a central wavelength of $1550 \mathrm{~nm}$. Every normalized efficiency is calculated from the average power of a SH pulse, which is equal to the total of the intensities at each frequency component in the spectrum. The oscillation with certain regularity appears on the curve and becomes particularly strong near $1550 \mathrm{~nm}$, as shown in the inset of Fig. 1(a). The highest conversion efficiency appears in an $11 \mathrm{~nm}$ region from 1545 to $1556 \mathrm{~nm}$. It can be seen that the highest efficiency would be achieved 


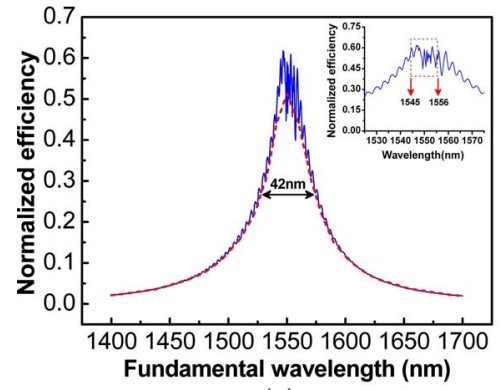

(a)

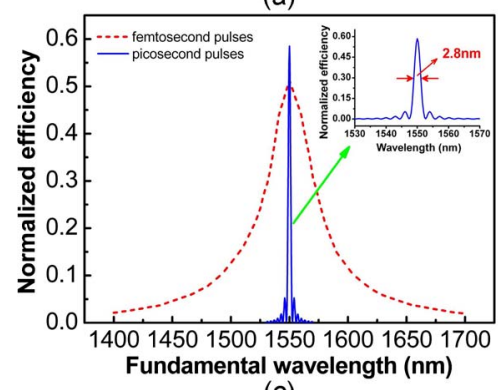

(c)

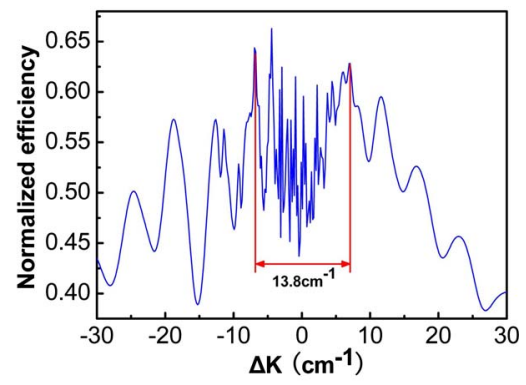

(b)

Fig. 1. (Color online) (a) Normalized SH efficiency as a function of pump pulse central wavelength. Inset, enlarged curve at the range from 1525 to $1575 \mathrm{~nm}$. (b) Efficiency with the phase mismatch. (c) Contrast picture of picosecond pulses' (solid curve) and femtosecond pulses' (dashed curve) wavelength tuning curve.

among an area close to the pulse central wavelength that matches the period. The low points of different parts in the tuning curve are joined, forming the dashed curve in Fig. 1(a), which is the bottom line $\mathrm{SH}$ efficiency can reach. The FWHM of it is as wide as 42 $\mathrm{nm}$. Figure 1(b) reveals the influence of the phase mismatch on the SHG efficiency. In a range of $13.8 \mathrm{~cm}^{-1}$ near $\Delta k=0$, the strong oscillation emerges and the left and right sides are not asymmetric. When the absolute value of $\Delta k$ increases, the efficiency variations decrease. Figure 1(c) shows the comparison of wavelength tuning curve between 40 ps and 40 fs pump pulses in SHG processes when other conditions remain the same. The inset of Fig. 1(c) presents a magnified wavelength tuning curve of 40 ps pump pulses with an FWHM of $2.8 \mathrm{~nm}$. Compared with the picosecond pulses, the femtosecond pulses are not necessarily superior in peak efficiency, but they have a much wider wavelength tuning range. For 40 fs pump pulses, this range is 15 times of that for 40 ps pulses. By utilizing a femtosecond laser source, highly efficient output of frequency doubling can be achieved in a much larger wavelength range.

In our experiment, a $10-\mathrm{mm}$-long and $0.5-\mathrm{mm}$ thick $Z$-cut PPLN sample with a period of $18.6 \mu \mathrm{m}$ was used for waveguide fabrication. The PPLN sample was made by the external pulse field poling technique. The $50 \mathrm{fs}$ waveguide writing pulses were from a Ti:sapphire femtosecond laser system operating at a central wavelength of $800 \mathrm{~nm}$ with a repetition of $1 \mathrm{KHz}$. The light was linearly polarized with polarization direction parallel to the waveguide axis and incident along the $z$ axis of the poled sample. The laser beam was focused into the sample by a $25 \times \mathrm{mi}-$ croscope objective $(\mathrm{NA}=0.4)$ and the focal point was approximately $200 \mu \mathrm{m}$ beneath the surface of the sample. To produce a thermally stable type II wave- guide, a pair of straight lines was written along the $X$ direction with a velocity of $400 \mu \mathrm{m} / \mathrm{s}$, and the line spacing was $10 \mu \mathrm{m}$. The single pulse energy was adjusted to $10 \mu \mathrm{J}$. It is found from our investigation that only the light polarized along the $Z$ axis [transverse-magnetic (TM) polarization] is guided in the laser-written lines.

The experimental arrangement of femtosecond pulses SHG (shown in Fig. 2) consisted of a femtosecond pulse laser system, which can generate $40 \mathrm{fs}$ pulses over a tunable wavelength range of 1160-2600 $\mathrm{nm}$ at a repetition rate of $1 \mathrm{KHz}$. The PPLN waveguide was mounted on a temperature-controlled oven. A $10 \times$ microscope objective $(\mathrm{NA}=0.25)$ was used to couple the laser beam into the PPLN waveguide.

A coupling loss of $1 \mathrm{~dB} /$ facet and a propagation loss of $0.8 \mathrm{~dB} / \mathrm{cm}$ were measured at $1550 \mathrm{~nm}$ for TM polarization. The input spectral width is $75.4 \mathrm{~nm}$, as depicted in Fig. 3(a). From Fig. 3(b), it can be seen that there exists a good agreement between experimental and theoretical data. The measured $\mathrm{SH}$ spectral width is $4.2 \mathrm{~nm}$ and the maximum point can be found at $774.6 \mathrm{~nm}$. Corresponding to the fundamental spectrum, the width of SH spectrum is so narrow because of the large group-velocity mismatch [9]. The walk-off

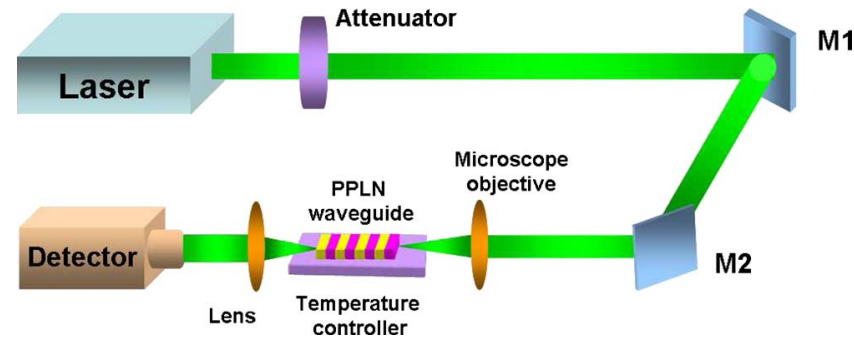

Fig. 2. (Color online) Experimental arrangement of SHG in the waveguide. 


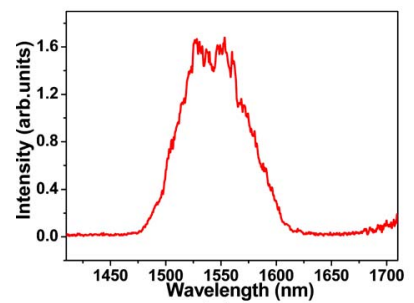

(a)

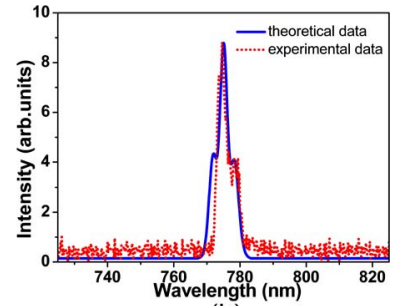

(b)
Fig. 3. (Color online) (a) Input spectrum, (b) SH spectrum of experimental (dotted curve) and theoretical (solid curve) results.

length is only about $0.1 \mathrm{~mm}$, while the SHG interaction length is $1 \mathrm{~cm}$. In addition to the main peak, two pronounced spectral dips appear on both sides of the spectrum. Moreover, the asymmetry of the two dips in experimental spectrum is more obvious than that of the calculated spectrum. The asymmetry of the spectrum may be caused by strong nonlinear effects owing to such high-peak power of femtosecond pulse, or a nonuniform temperature distribution in the temperature-controlling oven [10]. The near-field mode profile of SH pulses is shown in Fig. 4. The NA of the waveguide calculated from an estimated refractive index change of $\Delta n \approx 3 \times 10^{-3}$ is about 0.166 .

Figure 5 shows the experimental data of $\mathrm{SH}$ power with the input power. In the experiment, $\mathrm{SH}$ power increases almost linearly as the input power increases. In our simulation, the curve fitting shows the trend will be quadratic as the input power is enhanced. Therefore, we estimate if the input power is increased to a higher range, the curve would follow a quadratic trend. At an incident average power of 26.8 $\mathrm{mW}$, the maximum conversion efficiency of $18.2 \%$ is obtained. According to $\bar{\eta}=P_{2 \omega} / P_{\omega}^{2} L^{2}(L$ is the length of PPLN waveguide), we get the normalized efficiency of $12.6 \% \mathrm{~W}^{-1} \mathrm{~cm}^{-2}$, which is almost twice of the best published results [5].

In summary, we have investigated the tuning characteristics of ultrashort pulses, and we have demonstrated by experiments on frequency doubling of $40 \mathrm{fs}$ pulses in a 10-mm-long double-line-written PPLN waveguide.

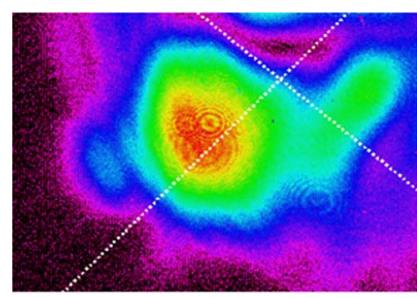

(a)

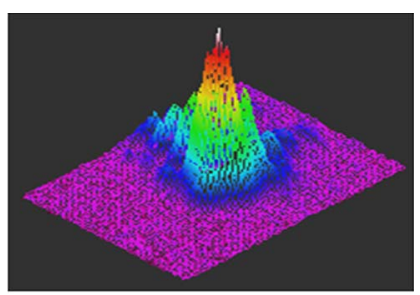

(b)
Fig. 4. (Color online) (a) 2D image of near field mode at $\mathrm{SH}$ wavelength, (b) corresponding $3 \mathrm{D}$ image.

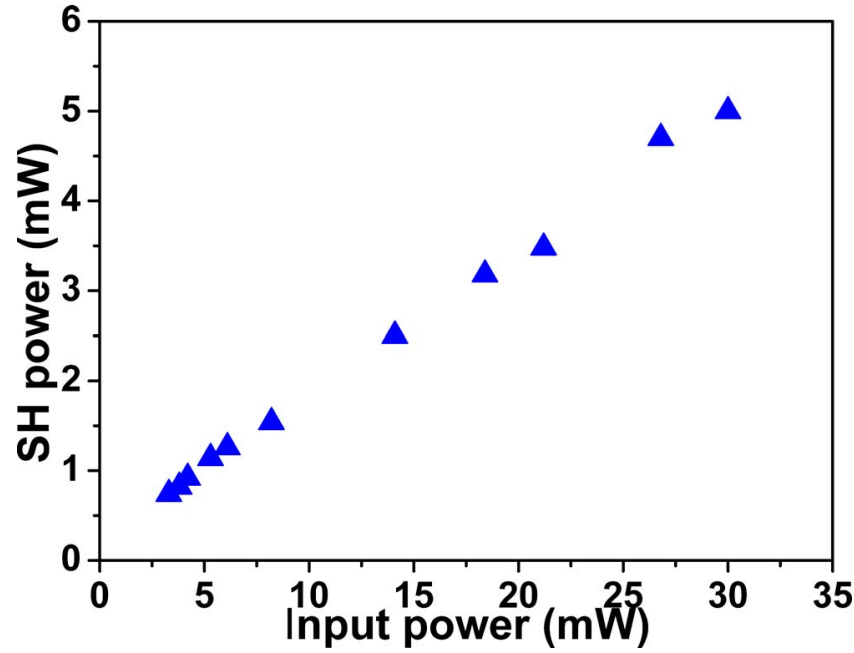

Fig. 5. (Color online) $\mathrm{SH}$ power versus fundamental power.

This work was supported by the National Natural Science Foundation of China (NSFC) (60677013, 60808003), the Specialized Research Fund for the Doctoral Program Foundation of Institute of Higher Education of China (20060055021, 20090031120004), and the MOE Key Lab of ATF-WL-NPM, Nankai University, Tianjin 300457, China.

\section{References}

1. K. M. Davis, K. Miura, N. Sugimoto, and K. Hirao, Opt. Lett. 21, 1729 (1996).

2. R. R. Thomson, S. Campbell, I. J. Blewitt, A. K. Kar, and D. T. Reid, Appl. Phys. Lett. 88, 111109 (2006).

3. Y. L. Lee, N. E. Yu, C. Jung, B. A. Yu, I. B. Sohn, S. C. Choi, Y. C. Noh, D. K. Ko, W. S. Yang, H. M. Lee, W. K. Kim, and H. Y. Lee, Appl. Phys. Lett. 89, 171103 (2006).

4. J. Burghoff, C. Grebing, S. Nolte, and A. Tünnermann, Appl. Phys. Lett. 89, 081108 (2006).

5. R. Osellame, M. Lobino, N. Chiodo, M. Marangoni, G. Cerullo, R. Ramponi, H. T. Bookey, R. R. Thomson, N. D. Psaila, and A. K. Kar, Appl. Phys. Lett. 90, 241107 (2007).

6. J. Thomas, M. Heinrich, J. Burghoff, S. Nolte, A. Ancona, and A. Tünnermann, Appl. Phys. Lett. 91, 151108 (2007).

7. S. Campbell, R. R. Thomson, D. P. Hand, A. K. Kar, D. T. Reid, C. Canalias, V. Pasiskevicius, and F. Laurell, Opt. Express 15, 17146 (2007).

8. Z. Zheng, A. M. Weiner, K. R. Parameswaran, M. H. Chou, and M. M. Fejer, J. Opt. Soc. Am. B 19, 839 (2002).

9. N. E. Yu, J. H. Ro, M. Cha, S. Kurimura, and T. Taira, Opt. Lett. 27, 1046 (2002).

10. R. Schiek, Y. Baek, and G. I. Stegeman, J. Opt. Soc. Am. B 15, 2255 (1998). 